\title{
华北地区胡桃楸林分布规律及群落构建机制分析
}

\author{
唐丽丽 ${ }^{1}$ 张 梅 ${ }^{1}$ 赵香林 $^{1}$ 康慕谊 $^{2,3}$ 刘鸿雁 $^{4}$ 高贤明 $^{5}$ 杨 泮 郑璞帆 $^{1}$ \\ 石福臣 ${ }^{1 *}$
}

${ }^{1}$ 南开大学生命科学学院, 天津 $300071{ }^{2}$ 北京师范大学地表过程与资源生态国家重点实验室, 北京 $100875 ;{ }^{3}$ 北京师范大学地理科学学部自然资源学 院, 北京 $100875 ;{ }^{4}$ 北京大学城市与环境学院, 北京大学生态研究中心, 地表过程分析与模拟教育部重点实验室, 北京 $100871 ;{ }^{5}$ 中国科学院植物研究 所植被与环境变化国家重点试验室, 北京 100093

\begin{abstract}
摘 要 探究植物分布规律和群落构建机制是揭示植物群落空间分布、群落物种多样性的形成、发展及其影响因素的重要途 径。该文以华北地区胡桃楸(Juglans mandshurica)林为研究对象, 基于野外 84 个样方的调查数据, 通过径级分析、典范对应分 析等方法研究了胡桃梑林的空间分布规律, 并通过亲缘关系指数计算、植物功能性状等方法研究了胡桃梑林的物种共存机 制。结果表明, 华北地区胡桃楸的胸径相对较小 (平均 $5.36 \mathrm{~cm}$ ), 种群年龄较低; 海拔、坡度、坡位和人为干扰程度是影响华 北地区胡桃楸分布的主要因子。研究区域内的胡桃楸大部分生长于山体中下部海拔较低的缓坡, 且人为干扰相对较少的区域, 各分布区域中胡桃梑林的分布规律各异。胡桃梑林物种的构建由生态位机制主导, 其中河北、陕西、天津的胡桃楸林物种共 存过程主要受负密度制约的影响, 北京、山西的胡桃楸林的物种共存过程主要受环境选择驱动。
\end{abstract}

关键词＼cjkstart胡桃梑；分布规律；谱系发育；群落构建机制；植物群落

唐丽丽, 张梅, 赵香林, 康慕谊, 刘鸿雁, 高贤明, 杨㔙, 郑璞帆, 石福臣 (2019). 华北地区胡桃楸林分布规律及群落构建机制分析. 植物生态学报, 43, 753-761. DOI: $10.17521 /$ cjpe.2018.0161

\section{Species distribution and community assembly rules of Juglans mandshurica in North China}

TANG Li-Li ${ }^{1}$, ZHANG Mei ${ }^{1}$, ZHAO Xiang-Lin ${ }^{1}$, KANG Mu-Yi ${ }^{2,3}$, LIU Hong-Yan ${ }^{4}$, GAO Xian-Ming ${ }^{5}$, YANG Tong ${ }^{1}$, ZHENG Pu-Fan ${ }^{1}$, and SHI Fu-Chen ${ }^{1 *}$

${ }^{1}$ College of Life Sciences, Nankai University, Tianjin 300071, China; ${ }^{2}$ State Key Laboratory of Earth Surface Processes and Resource Ecology, Beijing Normal University, Beijing 100875, China; ${ }^{3}$ College of Resources Science \& Technology, Faculty of Geographical Science, Beijing Normal University, Beijing 100875, China; ${ }^{4}$ Institute of Ecology, College of Urban and Environmental Sciences, Laboratory for Earth Surface Processes of the Ministry of Education, Peking University, Beijing 100871, China; and ${ }^{5}$ State Key Laboratory of Vegetation and Environmental Change, Institute of Botany, Chinese Academy of Sciences, Beijing 100093, China

\section{Abstract}

Aims Explore the distribution pattern and the processes controlling the assembly of the Juglans mandshurica dominated forests in North China.

Methods We investigated 84 plots, each with an area of $20 \mathrm{~m} \times 30 \mathrm{~m}$, for the $J$. mandshurica forests in the North China. We analyzed demography of the J. mandshurica based on its diameters, and applied canonical correspondence analysis (CCA) to explore the relationship between environment and species composition of different formations of the J. mandshurica forests. We then calculated phylogenetic index, i.e., net relatedness index (NRI) and nearest taxonomic index (NTI) and Gaussian Kernel Density Estimation (Gaussian KDE), for each plot to explore the rules controlling community assembly of these forests.

Important findings The population of $J$. mandshurica is relatively young in the Northern China, with a mean diameters at breast height $(D B H)$ of $5.36 \mathrm{~cm}$. Distribution of $J$. mandshurica in North China varied significantly with altitude, slope, slope position and human disturbance. Most of the J. mandshurica located at the low part of the massif with lower altitude and less human disturbance. In North China, niche related processes regulated species coexistence of Form. J. mandshurica, with competition controlled the community assembly in Hebei, Shaanxi and Tianjin, while environment filtering dominated in Beijing and Shanxi.

Key words Juglans mandshurica; distribution pattern; phylogeny; community assembly rules; plant community

Tang LL, Zhang M, Zhao XL, Kang MY, Liu HY, Gao XM, Yang T, Zheng PF, Shi FC (2019). Species distribution and community assembly rules of Juglans mandshurica in North China. Chinese Journal of Plant Ecology, 43, 753-761. DOI: 10.17521/cjpe.2018.0161 
群落构建机制即群落物种多样性形成及其维持 机制, 主要研究植物群落如何构建, 什么驱使植物 的多样性及群落功能的变化(Cavender-Bares et al., 2009)。目前对群落构建机制的分析主要基于谱系发 育和植物功能性状(Webb et al., 2002; Wright, 2004; Kraft et al., 2008; Swenson, 2011; 马克平, 2016; D’Amen, 2017), 对群落构建机制的解释包括生态 位机制(Diamond, 1975)和中性理论(Hubbell, 2001)。 中性理论认为群落内物种的出生、死亡、迁入、迁 出处于一个动态的平衡, 这种动态平衡维持着群落 内物种数量的稳定(Hubbell, 2001)。生态位机制则认 为群落内植物的生长繁殖、迁入迁出源自于群落的 生境、资源及生物间相互作用, 生态位机制是由环 境过滤和负密度制约(Bazzaz, 1991; Weiher \& Keddy, 1999)两个过程主导群落构建。

随着相关学科领域的快速发展, Webb等(2002) 提出用植物谱系发育理念来理解群落物种共存过程, 并提出净亲缘指数 $(N R I)$ 和最近亲缘指数 $(N T I)$ 这两 种亲缘关系指数, 以此来计算群落内物种间的系统 发育距离, 并在植物功能性状生态位保守和趋同两 种情况下讨论群落的构建机制(Kraft et al., 2007; Vamosi et al., 2009)。若亲缘关系指数大于 0 , 则物种 系统发育距离较小, 群落由亲缘关系较近的物种构 成; 若亲缘关系指数小于 0 , 说明物种系统发育距 离较大, 群落由亲缘关系较远的物种构成; 若亲缘 关系指数为 0 , 说明物种在系统发育结构上呈现随 机状态(Webb et al., 2002)。若群落物种生态位保守, 环境过滤作用会导致群落谱系结构的聚集, 竞争排 斥(负密度制约)作用会导致群落谱系结构的发散; 若群落物种生态位趋同, 环境过滤作用会导致群落 谱系结构发散, 负密度制约作用会导致群落谱系结 构随机或聚集(Webb et al., 2002; Kraft et al., 2007; Vamosi et al., 2009)。在中性作用下, 群落谱系结构 均呈随机状态(McPeek, 2007)。群落生态学与植物谱 系发育概念的结合, 为解释群落的构建提供了新的 理念。

胡桃楸 (Juglans mandshurica)又名核桃楸, 隶属 于胡桃科胡桃属(Juglans), 属第三纪子遗植物, 在 我国多分布于东北的东部山地。同时，胡桃楸的分布 区域也在向南延伸, 扩展到我国华北地区, 主要为 北京、天津等地的山区, 且颇具规模。目前针对东北 地区分布的胡桃楸种群的研究较为丰富全面(马万里
等, 2001; 梁淑娟等, 2005; 王东娜等, 2011; 王东亮, 2014; 苏金娟和王晓春，2017)，而华北地区胡桃楸 种群的研究相对较少, 且多集中在胡桃楸生理学特 性及群落结构等方面(间娜等, 2014; 高张莹等, 2017, Song et al., 2017)。华北地区胡桃楸种群的分布规 律、影响其分布的环境因子、胡桃楸林的系统发育 结构, 以及群落构建机制都尚未清楚。本文利用在 “华北地区自然植物群落资源综合考察”中获得的大 量胡桃楸样方数据, 对华北地区胡桃楸种群分布规 律及其影响因素进行分析, 并综合谱系发育和功能 性状探讨胡桃楸林的物种共存过程, 为胡桃楸林及 其生境的保护提供科学依据, 也为进一步探究华北 地区胡桃楸林物种组成及群落发展方向奠定基础。

\section{1 材料和方法}

\section{1 研究区域}

研究区域以华北地区为主，包括北京、天津、 河北、山西、陕西、河南6个省市(图1)。该区域背 依中国西北腹地, 南接长江中下游平原, 东临渤海。 研究区内地势西北高, 东南低, 海拔1-3 $691 \mathrm{~m}$, 地 形复杂多样, 包括平原、山地、高原、丘陵、盆地 等。气候以温带大陆性气候为主, 夏季高温多雨, 冬 季寒冷干旱, 年平均气温 $8-13{ }^{\circ} \mathrm{C}$, 年降水量 $400-$ $1000 \mathrm{~mm}$ 。研究区内生态系统类型丰富多样, 涵盖 山地森林、灌从、草原、湿地等。

\section{2 研究方法}

\subsection{1 野外调查及径级划分}

华北地区胡桃楸林主要为天然次生林, 本文采

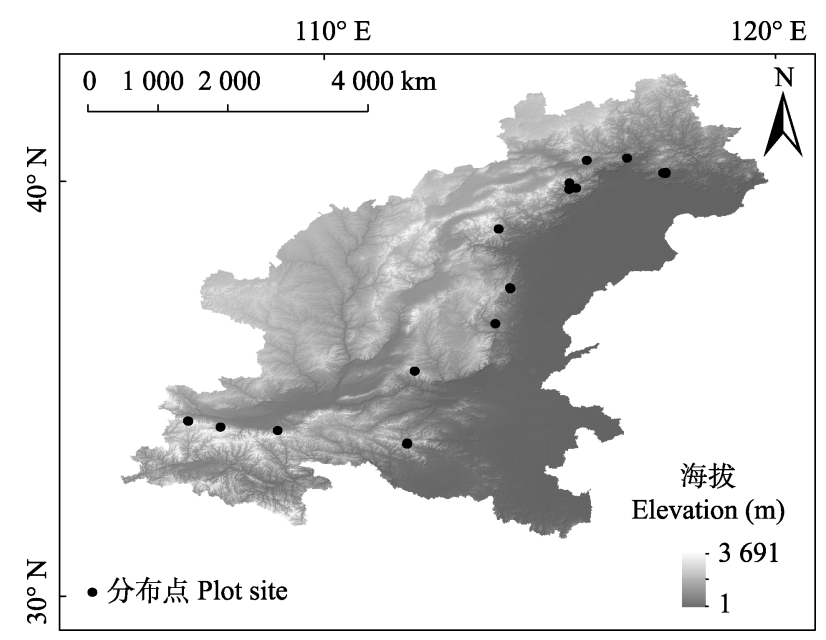

图1 华北地区胡桃楸样地分布。

Fig. 1 Sampling sites of Juglans mandshurica in North China. 
用样方法对其进行野外调查, 样方设置方法参照方 精云等(2009)的植物群落清查的技术规范, 样方尺 度主要为 $20 \mathrm{~m} \times 30 \mathrm{~m}$, 记录样方内所有胸径 $(D B H)$ $\geqslant 1 \mathrm{~cm}$ 的木本植物和所有草本植物的相关信息, 其 中木本植物主要记录种类、数量、树高、胸径, 草 本植物主要记录种类、多度、盖度, 同时记录样地 生境信息, 如样方坐标、海拔、坡度、坡向等信息。 根据各地胡桃楸种群分布及规模, 在研究区域内共 设 12 个样地，其中北京 3 个、天津 1 个、河北 2 个、山 西 2 个、陕西 3 个、河南 1 个, 12 个样地中共设有 84 个 样方(附录I), 样地具体分布位置如图1所示。根据相 关技术规范, 本研究采用 $2 \mathrm{~cm}$ 径距将胡桃楸胸径划 分为 9 个径级, 并结合其分布位点, 利用 ArcGIS 10.2 分析不同径级胡桃楸在华北地区的分布。

\subsection{2 胡桃楸空间分布及其影响因子分析}

根据样地调查结果及华北地区数字高程图(来 源于中国科学院资源环境科学数据中心), 利用 ArcGIS 10.2, 分析华北地区胡桃楸的空间分布规 律。使用典范对应分析(CCA)方法, 定量分析海拔、 坡度、坡向、坡位、人为干扰对胡桃楸在华北地区 分布的影响。其中坡向按照其度数分为北、东北、 西北、东、西、东南、西南、南, 分别用编号1-8代 表。坡位分为谷地、下、中下、中、中上和上 6 个坡 位, 编号1-6。人为干扰主要为伐木, 根据国家标准 生物多样性观测技术导则(HJ710.12-2016)中对人为 干扰活动的分类, 将人为干扰分为 4 个程度: 无干 扰指生境及植物群落没有受到人为干扰, 植被保持 原始状态, 对野生植物的生存繁衍没有任何影响, 在计算中赋值为 1 ; 轻微干扰指生境及植物群落受 到一定程度的影响, 但植被基本保持原始状态, 对 野生植物生存繁衍影响不大, 在计算中赋值为 2 ; 中度干扰指生境及植物群落受到干扰, 部分植被消 失, 但干扰消失后, 植被仍可恢复, 野生植物生存 繁衍受到一定程度的影响, 但仍然可以栖息繁衍, 在计算中赋值为 3 ; 重度干扰指生境及植物群落受 到严重干扰, 植被基本消失, 野生植物难以生存繁 衍, 在计算中赋值为4。此过程在R 3.3.1中的“vegan” 程序包(Jari et al., 2016)中进行。

\subsection{3 亲缘关系指数分析}

本文参考Webb等(2002)的实验方法构建系统发 育树。将样方调查中得到的种子植物, 按照科/属/ 种的格式, 输入到phylomatic平台(Webb \& Dong- oghue, 2005; Webb et al., 2008), 得到基于APG III分 类系统的具有进化枝长的系统发育树。根据各物种 在系统发育树上的枝长和所在样方中的数量, 计算 得出各样方的亲缘关系指数 (NRI和NTI)。此过程在 R 3.3.1 的“picante”程序包(Kembel et al., 2010)中进行。 因河南的胡桃楸数量较少, 仅在落叶柇林和华山松 林中零散分布, 因此并未对河南的胡桃楸数据进行 群落构建机制分析。

本文采用唐丽丽等(2017)对燕山东麓植物群落 构建机制的研究中提出的高斯核密度估计的方法对 亲缘关系指数的正负趋势进行分析。高斯核密度估 计方法就是利用高斯核密度估计来绘制出亲缘关系 指数的密度分布图, 并估算 $N R I 、 N T I$ 在正、负区域 内的面积, 若指数在正值区域面积大于其在负值区 域的面积, 则该指数趋近于正值, 反之则该指数 趋近于负值, 进而探究各群落谱系结构聚集、发散 还是随机。可视化及面积计算过程在 R 3.3.1 的 “ggplot2”和“zoo”程序包 (Achim \& Gabor, 2005; Wickham, 2016)中完成。

\subsection{4 系统发育信号检验}

本文采用Blomberg等(2003) 提出的 $K$ 值法, 以 植物最大树高作为功能性状, 检测胡桃楸林内的植 物系统发育信号。K值为实际的MSEO/MSE相对于其 期望值的比值, $M S E 0$ 是系统发育树末端物种性状数 据的均方误差, MSE是基于系统发育树的方差-协方 差矩阵计算的均方误差, 期望值 MSEO/MSE是基于 现有系统发育树的拓扑结构和枝长, 并假设性状以 布朗模型方式进化计算得出的。此外, 通过置换检 验来判断功能性状的系统发育信号是否显著 $(p<$ 0.05), 随机置换次数为 999 次。在 $\mathrm{R}$ 的 “ape” 和 “picante”程序包(Kembel et al., 2010; Paradis \& Schliep, 2019)中完成 $K$ 值的计算。若 $K<1$, 物种表现 出趋同性进化趋势; $K=1$, 物种随机进化; $K>1$, 物种进化与系统发育关系紧密, 功能性状保守。

\section{2 结果和分析}

\section{1 华北地区胡桃楸分布}

根据调查结果, 胡桃楸在华北地区分布范围较 广, 其分布海拔自 $320 \mathrm{~m}$ 至 $1700 \mathrm{~m}$, 纬度上跨越 $10^{\circ}$ 左右, 经度上跨越 $13^{\circ}$ 左右(图1)。根据调查结果, 胡 桃楸种群密度在河北最大, 河南最小(表1)。由图2 可知, 调查样方内胡桃楸的胸径普遍较小, 胸径为 
表1 华北地区各地核桃楸种群密度及各径级胡桃楸数量

Table 1 Juglans mandshurica's population density and the numbers of each diameter classes in North China

\begin{tabular}{lrrrrrc}
\hline $\begin{array}{l}\text { 径级 } \\
\text { Diameter classes } \\
(\mathrm{cm})\end{array}$ & $\begin{array}{c}\text { 天津 } \\
\text { Tianjin }\end{array}$ & $\begin{array}{c}\text { 北京 } \\
\text { Beijing }\end{array}$ & $\begin{array}{c}\text { 河北 } \\
\text { Hebei }\end{array}$ & $\begin{array}{c}\text { 山西 } \\
\text { Shanxi }\end{array}$ & $\begin{array}{c}\text { 河南 } \\
\text { Henan }\end{array}$ & $\begin{array}{c}\text { 陕西 } \\
\text { Shaanxi }\end{array}$ \\
\hline $1-3$ & 109 & 12 & 17 & 7 & 11 & 18 \\
$3-5$ & 58 & 38 & 110 & 44 & 1 & 3 \\
$5-7$ & 90 & 30 & 0 & 46 & 1 & 1 \\
$7-9$ & 104 & 10 & 0 & 22 & 0 & 1 \\
$9-11$ & 56 & 5 & 0 & 2 & 0 & 3 \\
$11-13$ & 11 & 5 & 0 & 1 & 0 & 0 \\
$13-15$ & 5 & 0 & 0 & 0 & 0 & 3 \\
$15-17$ & 0 & 0 & 0 & 0 & 0 & 2 \\
$>17$ & 1 & 0 & 0 & 0 & 0 & 0
\end{tabular}

种群密度

$\begin{array}{lllllll}\text { Population density } & 14 & 25 & 53 & 23 & 6 & 9\end{array}$ (ind. $\cdot \mathrm{km}^{-2}$ )

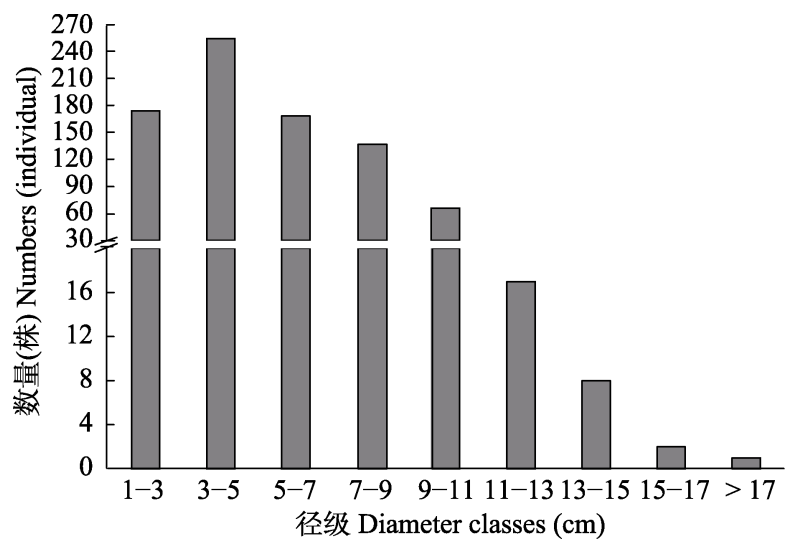

图2 华北地区胡桃楸胸径分级及其数量。

Fig. 2 Diameter classification and quantity of Juglans mandshurica in North China.

3-5 $\mathrm{cm}$ 的胡桃楸最多, 胸径大于 $11 \mathrm{~cm}$ 的胡桃楸数 量较少，而胸径为15-17 $\mathrm{cm}$ 的胡桃楸只有2株，17 $\mathrm{cm}$ 以上的仅有 1 株。由表 1 可知，天津的胡桃楸胸径 多样化程度最高, 其中胸径为 $1-3 \mathrm{~cm}$ 和 $7-9 \mathrm{~cm}$ 的胡 桃楸较多，而且调查样方内胸径最大 $(17.13 \mathrm{~cm})$ 的 胡桃楸也生长于天津; 河北胡桃楸的胸径多样性最 小, 大部分胸径集中在 3-5 $\mathrm{cm}$ 。山西和北京胡桃楸 的胸径多集中于 $3-7 \mathrm{~cm}$; 陕西胡桃楸的数量较少, 但胸径多样化程度相对较高, 其中胸径为 $1-3 \mathrm{~cm}$ 的 胡桃楸较多, 但胸径为 15-17 $\mathrm{cm}$ 的2株胡桃楸分布 于此。河南胡桃楸的数量最少, 其胸径多样化程度 较低, 胸径多为 $1-3 \mathrm{~cm}$ 。

\section{2 华北地区胡桃楸分布格局及其影响因子}

本文将胡桃楸种群分布情况与环境因子进行 $\mathrm{CCA}$ 分析, 模型变量的方差膨胀因子 $(V I F)$ 值为 7.56 ,
小于 10 , 说明模型中共线性不高, 模型可用。根据 CCA结果(表2)可知，前两轴与环境因子极显著相关 $(p<0.001)$, 相关系数分别为 $0.59 、 0.31$, 前两轴的方 差贡献率共为 $59.52 \%$ 。

根据前两轴种群分布-环境因子二维空间排序 (表2; 图3)可知，在CCA第一轴上，胡桃楸分布与海 拔极显著负相关，相关系数为 0.95 ; 与坡度极显著 正相关，相关系数为 0.57 ; 与坡位显著正相关，相 关系数为 0.45 ; 与人为干扰显著负相关，相关系数 为 0.17 , 即沿着第一轴从左到右, 海拔逐渐降低, 坡 度增大，坡位升高，人为干扰减弱。而对于 $\mathrm{CCA}$ 第 二轴上，坡度、坡位、人为干扰均分别与胡桃楸分

表2 华北地区胡桃楸分布与环境因子在前四排序轴上的相关系数 Table 2 Correlation coefficients between distribution of Juglans mandshurica and environmental factors at the first fourth canonical correspondence analysis (CCA) ordination axes in North China

\begin{tabular}{lcccc}
\hline 环境因子 & \multicolumn{4}{c}{ CCA排序轴 } \\
\cline { 2 - 5 } Environmental factor & CCA 1 & CCA 2 & CCA 3 & CCA 4 \\
\hline 海拔 Elevation & $-0.95^{* * *}$ & -0.06 & $-0.27^{*}$ & -0.02 \\
坡度 Slop & $0.57^{* * *}$ & $-0.54^{* * *}$ & $-0.39^{* * *}$ & $0.28^{*}$ \\
坡向 Aspect & 0.13 & 0.30 & $0.57^{* * *}$ & $-0.49^{* * *}$ \\
坡位 Slop position & $0.45^{* *}$ & $-0.48^{* * *}$ & $-0.37^{* * *}$ & $-0.64^{* * *}$ \\
人为干扰 Disturbance & $-0.17^{* *}$ & $-0.65^{* * *}$ & $0.68^{* * *}$ & -0.23 \\
特征值 Eigenvalue & $0.59^{* * *}$ & $0.31^{* * *}$ & $0.28^{* * *}$ & $0.20^{* * *}$ \\
累计贡献率 & & & & \\
Cumulative proportion & $39.08 \%$ & $59.52^{*} \%$ & $77.93 \%$ & $91.39 \%$ \\
\hline
\end{tabular}

*, $p<0.05 ;{ }^{* *}, p<0.01 ; * * *, p<0.001$

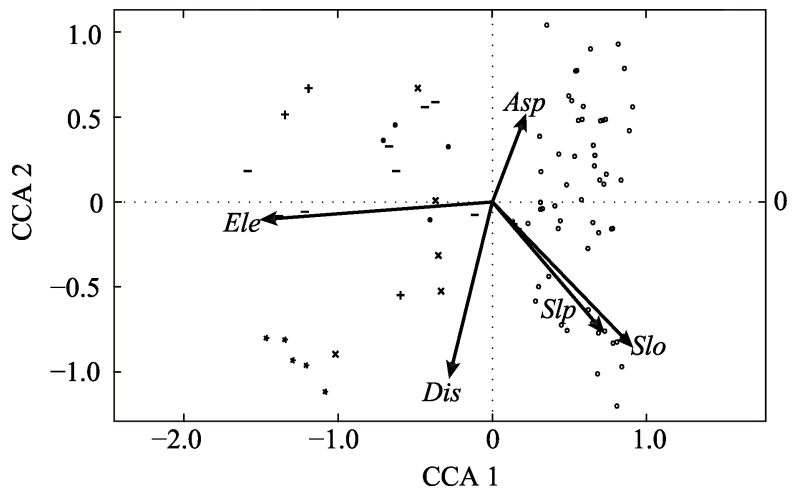

图3 华北地区胡桃楸分布与环境因子典范对应分析(CCA) 的二维排序图。Ele，海拔; Asp，坡向; Slo, 坡度; Slp, 坡位; Dis, 人为干扰。 $\times$, 北京; $\circ$, 天津; $\bullet$, 河北; *, 河南; + , 山西; -, 陕西。

Fig. 3 Two-dimensional ordination diagram of canonical correspondence analysis (CCA) between distribution of Juglans mandshurica and environmental factors in North China. Ele, elevation; Asp, aspect; Slo, slope; Slp, slop position; Dis, disturbance. $\times$, Beijing; ॰, Tianjin; •, Hebei; *, Henan; +, Shanxi; -, Shaanxi.

www.plant-ecology.com 
布呈显著负相关关系, 沿着第二轴从下到上坡度减 小、坡位降低, 人为干扰减弱。坡向在第一、第二 轴上相关性不显著, 但在第三轴上呈极显著正相关 关系, 在第四轴上呈极显著负相关关系, 因此模型 中也保留了坡向这一环境因子。

根据前两轴种群分布-环境因子二维空间排序 中胡桃楸的分布(图3)可知, 北京、天津、河北地区 的胡桃楸普遍分布在海拔较低、坡度较缓、人为干 扰较少的区域; 河南的胡桃楸则集中分布在高海 拔、人为干扰程度强的缓坡上; 山西、陕西地区的 胡桃楸分布在海拔上呈现出低、中、高 3 个水平。

\section{3 不同分布区域胡桃梑群落构建机制}

通过高斯核密度估计结果(表3; 图4)可知, 北 京的胡桃楸林 NRI、NTI趋近于负值, 河北的 NRI、 $N T I$ 趋近于正值, 山西的 $N R I 、 N T I$ 趋近于负值, 陕西 和天津的 $N R I 、 N T I$ 均趋近于正值。山西只有一个胡 桃楸样方, 其 $N R I$ 为 $-2.19, N T I$ 为 -4.10 , 无法估计其 整体趋势。

根据功能性状-最大树高的系统发育信号检测 结果 $(K=0.82, p=0.001)$ 可知, 这一功能性状检测 到了显著的系统发育信号 $(p<0.05)$, 群落物种的功 能性状表现出趋同性进化趋势。根据NRI、NTI值可 知, 河北、陕西地区胡桃楸林物种的系统发育结构 聚集 $(N R I>0, N T I>0)$ (图4), 群落由亲缘关系比较 密切的物种构成; 天津、北京地区的胡桃楸林物种 的系统发育结构有些复杂, 整体上天津呈现聚集 状态, 北京呈现发散状态, 但在天津也有部分胡桃
楸林的系统发育结构是发散的，在北京也有部分 胡桃楸林的发育结构是聚集的(NRI、NTI正负都 有)(图4)。

基于功能性状系统发育信号和亲缘关系指数, 可以认为整体上河北、陕西、天津地区胡桃楸林物 种共存是负密度制约作用驱动的，而北京、山西胡 桃楸林物种共存是环境选择作用驱动的。但对于天 津地区胡桃楸林也有部分可能受环境选择驱动物种 共存, 且在北京也有部分胡桃楸林受负密度制约驱 动物种共存。

\section{3 讨论}

\section{1 胡桃楸种群分布规律}

胡桃楸种群在我国主要分布在东北和华北地区, 东北地区主要集中在长白山和小兴安岭海拔200$1000 \mathrm{~m}$ 的阔叶林带和针阔混交林带(马万里等, 2008)。在东北长白山地区的胡桃楸种群呈现出衰退 型的年龄结构, 幼苗和幼树比例较小 (马万里等, 2008)。从华北地区的胡桃楸的胸径来看, 华北地区 的胡桃楸种群年龄较小, 幼苗、幼树较多(胸径集中 在11 cm以下)。这与胡桃楸林在东北、华北地区所 处的生境及森林发展历史的差异联系密切(赵光仪 等, 1991; 马万里等, 2005; 王建文, 2006)。胡桃楸属 第三纪子遗植物, 起源古老, 东北长白山和小兴安 岭地区是胡桃楸的最适生长区，相对而言，华北是 我国胡桃楸种群分布的南部边缘地区，苏金娟和王 晓春(2017)的研究表明胡桃楸的生长与气温呈现负
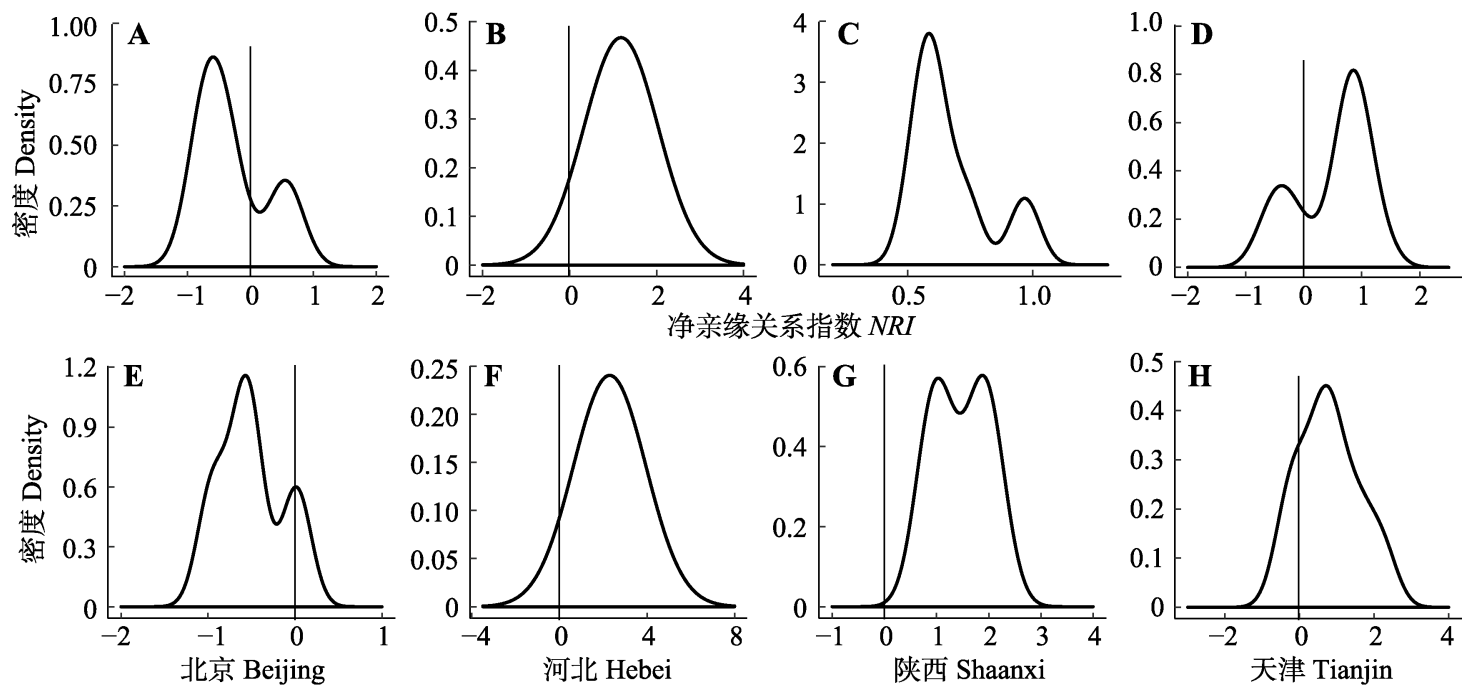

图4 亲缘关系指数高斯核密度估计曲线。NRI, 净亲缘关系指数; NTI, 最近亲缘关系指数.

Fig. 4 Results of Gaussian kernel density estimation of phylogenetic index. NRI, net relatedness index; NTI, nearest taxonomic index. 
表3 5 个省市净亲缘关系指数 $(N R I)$ 、最近亲缘关系指数 $(N T I)$ 在正负范 围内的面积

Table 3 Positive and negative area under curve of the net relatedness index $(N R I)$ and net nearest taxa index $(N T I)$ in five provinces

\begin{tabular}{ccccccc}
\hline & & $\begin{array}{c}\text { 北京 } \\
\text { Beijing }\end{array}$ & $\begin{array}{c}\text { 河北 } \\
\text { Hebei }\end{array}$ & $\begin{array}{c}\text { 山西 } \\
\text { Shanxi }\end{array}$ & $\begin{array}{c}\text { 陕西 } \\
\text { Shaanxi }\end{array}$ & $\begin{array}{c}\text { 天津 } \\
\text { Tianjin }\end{array}$ \\
\hline NRI & $<0$ & 0.72 & 0.08 & - & 0.00 & 0.26 \\
& $>0$ & 0.28 & 0.92 & - & 1.00 & 0.74 \\
\multirow{2}{*}{$N T I$} & $<0$ & 0.86 & 0.08 & - & 0.00 & 0.20 \\
& $>0$ & 0.13 & 0.92 & - & 1.00 & 0.80 \\
\hline
\end{tabular}

相关关系，因此在生境，尤其是生物气候方面，气 温较低的东北地区更适合胡桃楸生长, 其种群生长 速度比华北地区胡桃楸种群相对要快。另外, 在森 林发展历史方面, 东北地区森林历史悠久, 虽遭砍 伐, 但仍保有一定面积的原始森林, 其中不乏大量 的高龄树木(王建文, 2006)。而华北地区因人口众多, 迫于经济等压力, 在新中国成立前此地区的原始森 林均被破坏, 当前所看到的森林是新中国成立后通 过植树造林、森林保育等措施培育的人工林和衍生 的次生林, 因此华北地区成林历史较短, 其树木年 龄相对较小(王建文, 2006)。

\section{2 影响胡桃楸种群分布的因素}

根据本研究结果, 华北地区的胡桃楸种群主要 分布在燕山山脉海拔较低, 且人为干扰相对较小的 山体中下部缓坡区域。海拔、坡度、坡位及人为干 扰是影响华北地区胡桃楸种群分布的主要因子。 $\mathrm{CCA}$ 分析结果中环境因子前两轴的方差贡献率为 $59.52 \%$, 说明还存在其他影响因子, 如小气候和土 壤等都是影响植物分布的重要因素(Condit et al., 2013; Jones, 2013)。在华北地区森林群落和环境因 子关系的研究中曾发现土壤的相关指标是影响森林 群落分布的重要因素(余敏等, 2013; 白晓航等, 2017)。小气候和土壤因子对华北地区胡桃楸种群分 布的影响有待进一步研究。

\section{3 不同分布区域胡桃楸林物种共存过程的差异}

本研究中华北地区各分布区域的胡桃楸物种共 存过程有所不同, 这与各区域内胡桃楸林所处的环 境及其自身生长阶段有一定的关系。河北、陕西、 天津地区胡桃楸多分布于山体中部、人为干扰程度 较小、坡度较缓、海拔较低的生境, 这3个地区的生 境适宜植物生长, 环境条件并不是限制物种生存的 最大因素, 因此环境选择作用并不是胡桃楸林物种 共存的主要驱动因子; 而且这 3 个地区胡桃楸种群
密度较大，且胸径在1-3 cm的胡桃楸幼苗极多，植 物群落中种子和幼苗的生长易受其母体成树的限制 (Hubbell, 1980; Connell et al., 1984; Gilbert et al., 1994; Hyatt et al., 2003; 祝燕等, 2009), 因此在这3 个地区胡桃楸林的物种共存中起主要驱动作用的是 负密度制约作用。相对而言, 北京、山西胡桃楸所 处生境的山体坡度、坡位、人为干扰的梯度较大，生 境异质性高, 环境在植物生长共存过程中发挥更大 作用。而且北京、山西的胡桃楸林多为胸径 $3-7 \mathrm{~cm}$ 的幼树, 在此生长阶段, 母体成树对其的限制作用 会有所减弱(祝燕等, 2009)。环境条件和自身生长阶 段决定了北京、山西的胡桃楸林物种共存过程主要 受环境选择作用驱动。

另外, 天津部分胡桃楸林物种共存过程由环境 选择作用主导, 北京也有部分群落物种共存由负密 度制约作用主导。其原因在于, 首先天津也有部分 胸径较大的胡桃楸林存在, 很有可能随着幼苗的长 大，母体成树对其的限制作用在减弱(Connell et al., 1984; Condit et al., 2000; Wright, 2002), 负密度制约 作用也随之降低; 其次天津地区部分胡桃楸林的人 为干扰程度较强, 且处在坡度较陡的山体中上部, 生存环境较为恶劣, 因此环境选择作用主导这部分 群落中的物种共存过程。北京的胡桃楸林中也有部 分是胸径为 $1-3 \mathrm{~cm}$ 的幼苗, 且北京地区部分胡桃楸 林所处环境海拔相对较低, 人为干扰较少, 在这部 分胡桃楸林中环境可能不是其主要限制因子, 而是 负密度制约作用驱动着物种共存。

\section{4 结论}

本文对华北地区胡桃楸林的分布规律及其影响 因素、群落物种共存过程进行了系统分析。研究发 现受森林发展历史等因素的影响, 华北地区的胡桃 楸林种群年龄结构较低, 其分布也受众多生物、非 生物因子的影响，低山、缓坡、人为干扰较少的生 境是大多胡桃楸种群的分布区。生态位机制主导华 北地区胡桃楸林中群落结构及物种组成, 受所处的 环境及胡桃楸林自身发展阶段的影响, 环境选择和 负密度制约作用在各地发挥不同作用。经比较发现, 在样本量较大, 且群落环境较为复杂的研究中, 高 斯核密度估计方法能较为直接地揭示出亲缘关系指 数的正负趋势, 能够更为全面地判断群落中物种的 亲缘关系。 
致谢 感谢承担“华北地区自然植物群落资源综合 考察”项目的北京师范大学康慕谊、北京大学刘鸿 雁、中国科学院植物研究所高贤明、西北大学岳明、 山东大学王仁卿等课题组的项目参与人员, 在获取 本研究样方数据中付出的辛勤劳动。感谢天津市农 业农村委员会项目 (ITTFPRS2018001)和天津市科 学技术委员会项目(18ZXZYNC00120)资助。

\section{参考文献}

Bai XH, Zhang JT, Cao K, Wang YQ, Sadia S, Cao G (2017). Relationship between forest communities and the environment in the Xiaowutai Mountain National Nature Reserve, Hebei. Acta Ecologica Sinica, 37, 3683-3696. [白 晓航, 张金屯, 曹科, 王云泉, Sehrish Sadia, 曹格 (2017). 河北小五台山国家级自然保护区森林群落与环 境的关系. 生态学报, 37, 3683-3696.]

Bazzaz FA (1991). Habitat selection in plants. The American Naturalist, 137, S116-S130.

Blomberg SP, Garland T, Ives AR (2003). Testing for phylogenetic signal in comparative data: Behavioral traits are more labile. Evolution, 57, 717-745.

Cavender-Bares J, Kozak KH, Fine PVA, Kembel SW (2009). The merging of community ecology and phylogenetic biology. Ecology Letters, 12, 693-715.

Condit R, Ashton PS, Baker P, Bunyavejchewin S, Gunatilleke S, Gunatilleke N, Hubbell SP, Foster RB, Itoh A, LaFrankie JV, Lee HS, Losos E, Manokaran N, Sukumar R, Yamakura T (2000). Spatial patterns in the distribution of tropical tree species. Science, 288, 1414-1418.

Condit R, Engelbrecht BMJ, Pino D, Pérez R, Turner BL (2013). Species distributions in response to individual soil nutrients and seasonal drought across a community of tropical trees. Proceedings of the National Academy of Sciences of the United States of America, 110, 5064-5068.

Connell JH, Tracey JG, Webb LJ (1984). Compensatory recruitment, growth, and mortality as factors maintaining rain forest tree diversity. Ecological Monographs, 54, 141-164.

D'Amen M, Rahbek C, Zimmermann NE, Guisan A (2017). Spatial predictions at the community level: From current approaches to future frameworks. Biological Reviews, 92, 169-187.

Diamond JM (1975). Assembly of Species Communities. Harvard University Press, Boston. 342-344.

Fang JY, Wang XP, Shen ZH, Tang ZY, He JS, Yu D, Jiang Y, Wang ZH, Zheng CY, Zhu JL, Guo ZD (2009). Methods and protocols for plant community inventory. Biodiversity Science, 17，533-548. [方精云, 王襄平, 沈泽吴, 唐志 尧, 贺金生, 于丹, 江源, 王志恒, 郑成洋, 朱江玲, 郭 兆迪 (2009). 植物群落清查的主要内容、方法和技术规

\section{范. 生物多样性, 17, 533-548.]}

Gao ZY, Zhang HF, Chen GP, Feng XM, Zhao TJ, Gao X, Shi FC (2017). Fruit stone morphology and geographic variation in Juglans mandshurica populations. Chinese Journal of Applied and Environmental Biology, 23, 609-615. [高 张荣, 张海峰, 陈国平, 冯小梅, 赵铁建, 高金, 石福臣 (2017). 核桃楸种群果核形态及地理变异. 应用与环境 生物学报, 23, 609-615.]

Gilbert GS, Foster RB, Hubbell SP (1994). Density and distanceto-adult effects of a canker disease of trees in a moist tropical forest. Oecologia, 98, 100-108.

Hubbell SP (1980). Seed predation and the coexistence of tree species in tropical forests. Oikos, 35, 214-229.

Hubbell SP (2001). The Unified Neutral Theory of Biodiversity and Biogeography. Princeton University Press, Princeton.

Hyatt LA, Rosenberg MS, Howard TG, Bole G, Fang W, Anastasia J, Brown K, Grella R, Hinman K, Kurdziel JP, Gurevitch J (2003). The distance dependence prediction of the Janzen-Connell hypothesis: A meta-analysis. Oikos, 103, 590-602.

Jari O, Blanchet FG, Roeland K, Pierre L, Peter RM, O'Hara RB, Gavin LS, Peter S, Stevens MHH, Helene W (2016). Vegan: Community Ecology Package. R package version: 2.4-1. http://cran.r-project.org/package=vegan. Cited: 2018-07-07

Jones HG (2013). Plants and Microclimate: A Quantitative Approach to Environmental Plant Physiology. Cambridge University Press, Cambridge, UK.

Kembel SW, Cowan PD, Helmus MR, Cornwell WK, Morlon H, Ackerly DD, Blomberg SP, Webb CO (2010). Picante: $\mathrm{R}$ tools for integrating phylogenies and ecology. Bioinformatics, 26, 1463-1464.

Kraft NJB, Cornwell WK, Webb CO, Ackerly DD (2007). Trait evolution, community assembly, and the phylogenetic structure of ecological communities. The American Naturalist, 170, 271-283.

Kraft NJB, Valencia R, Ackerly DD (2008). Functional traits and niche-based tree community assembly in an Amazonian forest. Science, 322, 580-582.

Liang SJ, Pan P, Sun ZH, Wang QC (2005). Influence of slope on the growth of Fraxinus mandshurica Rup. and Juglans manshurica Maxim. plantations. Journal of Northeast Forestry University, 33(3), 18-19. [梁淑娟, 潘攀, 孙志 虎, 王庆成 (2005). 坡位对水曲柳及胡桃楸生长的影 响. 东北林业大学学报, 33(3), 18-19.]

Ma KP (2016). Hot topics for biodiversity science. Biodiversity Science, 24, 1-2. [马克平 (2016). 生物多样性科学的热 点问题. 生物多样性, 24, 1-2.]

Ma WL, Jing T, Kujansuu J, Luo JC, Sun B, Wang GF (2001). The dynamics of seed rain and seed bank of Juglans mandshurica population in the Changbai Mountain. Journal of Beijing Forestry University, 23(3), 70-72. [马万里, 荆涛, Kujansuu J, 罗菊春, 孙波, 王广发 (2001). 长白 
山地区胡桃楸种群的种子雨和种子库动态. 北京林业 大学学报, 23(3), 70-72.]

Ma WL, Luo JC, Jing T, Kujansuu J (2005). Ecological studies and prospect of cultivation on Juglans mandshurica population. Journal of Inner Mongolia Normal University (Natural Science Edition), 34, 489-492. [马万里, 罗菊春, 荆涛, Kujansuu J (2005). 珍贵树种核桃楸的生态学问题 及培育前景. 内蒙古师范大学学报 (自然科学汉文版), 34, 489-492.]

Ma WL, Luo JC, Jing T, Kujansuu J (2008). Study on dynamics of Juglans mandshurica population from Changbai Mountain. Bulletin of Botanical Research, 28, 249-253. [马万里, 罗菊春, 荆涛, Kujansuu J (2008). 长白山林区 核桃楸种群数量动态变化的研究. 植物研究, 28, 249-253.]

McPeek MA (2007). The macroevolutionary consequences of ecological differences among species. Palaeontology, 50, 111-129.

Paradis E, Schliep K (201P). ape 5.0: An environment for modern phylogenetics and evolutionary analyses in R. Bioinformatics, 35, 526-528.

Song NQ, Zhang JT, Zhao FG (2017). The PCA index for measuring functional diversity and its application to Juglans mandshurica communities in the Beijing mountains, China. International Journal of Biomathematics, 10, 1750097. DOI: $10.1142 / \mathrm{S} 1793524517500978$.

Su JJ, Wang XC (2017). Spatio-temporal variations in climategrowth relationships of three hardwood tree species across the north Zhangguangcai Mountains, northeast China. Acta Ecologica Sinica, 37，1484-1495. [苏金娟，王晓春 (2017). 张广才岭北部三大硬阔树木生长-气候关系的 时空变异. 生态学报, 37, 1484-1495.]

Swenson NG (2011). The role of evolutionary processes in producing biodiversity patterns, and the interrelationships between taxonomic, functional and phylogenetic biodiversity. American Journal of Botany, 98, 472-480.

Tang LL, Chen GP, Feng XM, Zhao TJ, Shi FC (2017). Community assembly rules of the east of Yanshan Mountain based on phylogeny. Bulletin of Botanical Research, 37, 807-815. [唐丽丽, 陈国平, 冯小梅, 赵铁建, 石福臣 (2017). 基于系统发育的燕山东麓植物群落的构建机制. 植物研究, 37, 807-815.]

Vamosi SM, Heard SB, Vamosi JC, Webb CO (2009). Emerging patterns in the comparative analysis of phylogenetic community structure. Molecular Ecology, 18, 572-592.

Wang DL (2014). The Research on the Interspecies Allelopathic Effect of Juglans mandshurica and Larix gmelinii Forest Plantation. Master degree dissertation, Northeast Forestry University, Harbin. [王东亮 (2014). 胡桃楸-落 叶松林地种间化感效应的研究. 硕士学位论文, 东北林 业大学, 哈尔滨.]

Wang DN, Mu CC, Gao Z, Feng FJ (2011). ISSR analysis of genetic diversity of Juglans mandshurica Maxim. populations. Nonwood Forest Research, 29(2), 22-29. [王东娜, 牟长城, 高卓, 冯福娟 (2011). 胡桃楸天然种群遗传多 样性的ISSR分析. 经济林研究, 29(2), 22-29.]

Wang JW (2006). A Study on the Historical Change of Foreset|Grassland and Eco-catastrophes in North China. $\mathrm{PhD}$ dissertation, Beijing Forestry University, Beijing. [王建文 (2006). 中国北方地区森林、草原变迁和生态灾害的历 史研究. 博士学位论文, 北京林业大学, 北京.]

Webb CO, Ackerly DD, Kembel SW (2008). Phylocom: Software for the analysis of phylogenetic community structure and trait evolution. Bioinformatics, 24, 2098-2100.

Webb CO, Ackerly DD, McPeek MA, Donoghue MJ (2002). Phylogenies and community ecology. Annual Review of Ecology and Systematics, 33, 475-505.

Webb CO, Donoghue MJ (2005). Phylomatic: Tree assembly for applied phylogenetics. Molecular Ecology Notes, 5, 181-183.

Weiher E, Keddy P (1999). Ecological Assembly Rules: Perspectives, Advances, Retreats. Cambridge University Press, Cambridge, UK.

Wickham H (2016). ggplot2: Elegant Graphics for Data Analysis. Springer, New York.

Wright IJ, Reich PB, Westoby M, Ackerly DD, Baruch Z, Bongers F, Cavender-Bares J, Chapin T, Cornelissen JHC, Diemer M, Flexas J, Garnier E, Groom PK, Gulias J, Hikosaka K, Lamont BB, Lee T, Lee W, Lusk C, Midgley JJ, Navas ML, Niinemets Ü, Oleksyn J, Osada N, Poorter H, Poot P, Prior L, Pyankov VI, Roumet C, Thomas SC, Tjoelker MG, Veneklaas EJ, Villar R (2004). The worldwide leaf economics spectrum. Nature, 428, 821-827.

Wright SJ (2002). Plant diversity in tropical forests: A review of mechanisms of species coexistence. Oecologia, 130, $1-14$.

Yan N, Chen T, Chen YL, Wu XL, Wu LJ, Dawa CM, Basang DJ, Xu JY (2014). Characteristics of population structure Juglans mandshurica from the Wuling Mountain Nature Reserve. Journal of Capital Normal University (Natural Sciences Edition), 35(6), 64-67, 81. [闵娜, 陈朋, 陈云 丽，吴晓丽，吴丽娟，达瓦措姆，巴桑多吉，徐建英 (2014). 雾灵山自然保护区胡桃楸种群结构特征分析. 首都师范大学学报(自然科学版), 35(6), 64-67, 81.]

Yu M, Zhou ZY, Kang FF, Ouyang S, Mi XC, Sun JX (2013). Gradient analysis and environmental interpretation of understory herb-layer communities in Xiaoshegou of Lingkong Mountain, Shanxi, China. Chinese Journal of Plant Ecology, 37, 373-383. [余敏, 周志勇, 康峰峰, 欧 阳帅, 米湘成, 孙建新 (2013). 山西灵空山小蛇沟林下 草本层植物群落梯度分析及环境解释. 植物生态学报, 37, 373-383.]

Zeileis A, Grothendieck G (2005). Zoo: S3 infrastructure for regular and irregular time series. Journal of Statistical

www.plant-ecology.com 
Software, 14, 1-27.

Zhao GY, Tian XJ, Wu ZH (1991). Analysis and discussion on the north distributing limitation of amur corktree, manchurian walnut and manchurian ash. Journal of Northest Forestry University, 19(Suppl. 1), 290-295. [赵光仪, 田兴军, 吴振河 (1991). 黄波罗、胡桃楸、水曲柳分布北限论析. 东北林业大学学报, 19(Suppl. 1), 290-295.]
Zhu Y, Mi XC, Ma KP (2009). A mechanism of plant species coexistence: The negative density-dependent hypothesis. Biodiversity Science, 17, 594-604. [祝燕, 米湘成，马克 平 (2009). 植物群落物种共存机制: 负密度制约假说. 生物多样性, 17, 594-604.]

责任编委: 唐志尧 责任编辑: 李 敏 实习编辑: 赵 航

\section{附录I 华北地区胡桃楸样方信息}

Supplement I The plots of Juglans mandshurica in North China

http://www.plant-ecology.com/fileup/1005-264X/PDF/cjpe.2018.0161-S1.pdf 
唐丽丽, 张梅, 赵香林, 康慕谊, 刘鸿雁, 高贤明, 杨粀, 郑璞帆, 石福臣 (2019). 华北地区 胡桃楸林分布规律及群落构建机制分析. 植物生态学报，43，753-761. DOI: 10.17521/cjpe.2018.0161

Tang LL, Zhang M, Zhao XL, Kang MY, Liu HY, Gao XM, Yang T, Zheng PF, Shi FC (2019). Species distribution and community assembly rules of Juglans mandshurica in North China. Chinese Journal of Plant Ecology, 43, 753-761. DOI: 10.17521/cjpe.2018.0161 http://www.plant-ecology.com/CN/10.17521/cjpe.2018.0161

附录I 华北地区胡桃楸样方信息

Supplement I The plots the Juglans mandshurica in North China

\begin{tabular}{|c|c|c|c|c|c|c|}
\hline 样地 & 核桃楸胸径 & 核桃梑个数 & 海拔 & 坡度 & 坡向 & 乔木层盖度 \\
\hline Plot & Diameter $(\mathrm{cm})$ & Number & Elevation (m) & Slope & Aspect & Coverage \\
\hline BEJ1 & 4.71 & 51 & 1220 & 31 & 320 & 85 \\
\hline BEJ2 & 6.18 & 35 & 1187 & 6 & 26 & 9 \\
\hline BEJ3 & 7.32 & 10 & 1243 & 19 & 158 & 85 \\
\hline BEJ4 & 4.75 & 8 & 795 & 10 & 275 & I \\
\hline BEJ5 & 7.58 & 3 & 936 & 20 & 190 & I \\
\hline HEB 1 & 3.79 & 42 & 1330 & 30 & 28 & 90 \\
\hline HEB2 & 3.57 & 39 & 1280 & 15 & 32 & 85 \\
\hline HEB3 & 3.66 & 45 & 1340 & 17 & 32 & 80 \\
\hline HEB4 & 2.13 & 1 & 906 & 8 & 0 & 50 \\
\hline SAX1 & 8.60 & 2 & 1318 & 28 & 45 & 85 \\
\hline SAX2 & 3.41 & 2 & 1123 & 30 & 30 & 90 \\
\hline SAX3 & 13.03 & 8 & 1669 & 5 & 350 & 78 \\
\hline SAX4 & 2.01 & 24 & 1587 & 10 & 220 & 80 \\
\hline SHX1 & 8.79 & 3 & 1698 & 0 & 35 & 85 \\
\hline SHX2 & 6.15 & 23 & 1611 & 10 & 0 & 40 \\
\hline SHX3 & 5.10 & 19 & 1611 & 5 & 0 & 35 \\
\hline SHX4 & 5.13 & 20 & 1222 & 10 & 90 & 70 \\
\hline SHX5 & 5.10 & 23 & 1226 & 13 & 100 & 75 \\
\hline SHX6 & 6.27 & 17 & 1165 & 5 & 330 & 60 \\
\hline SHX7 & 6.34 & 13 & 1164 & 10 & 320 & 75 \\
\hline SHX8 & 2.90 & 2 & 1062 & 32 & 0 & 85 \\
\hline SHX9 & 3.38 & 6 & 1057 & 35 & 350 & 80 \\
\hline HN1 & 2.04 & 1 & 1557 & 5 & 125 & 80 \\
\hline HN2 & 2.58 & 7 & 1530 & 5 & 80 & 85 \\
\hline HN3 & 2.10 & 7 & 1540 & 5 & 225 & 85 \\
\hline HN4 & 1.37 & 1 & 1486 & 12 & 190 & 85 \\
\hline HN5 & 2.80 & 1 & 1493 & 4 & 18 & 90 \\
\hline TJ1 & 4.90 & 8 & 500 & 15 & 270 & 85 \\
\hline $\mathrm{TJ} 2$ & 5.86 & 38 & 550 & 18 & 250 & 80 \\
\hline TJ3 & 1.91 & 1 & 445 & 22.5 & 270 & 70 \\
\hline TJ4 & 7.17 & 6 & 1000 & 30 & 70 & 90 \\
\hline TJ5 & 7.87 & 7 & 960 & 20 & 10 & 95 \\
\hline TJ6 & 9.30 & 4 & 960 & 36 & 100 & 95 \\
\hline TJ7 & 4.59 & 7 & 900 & 20 & 85 & 95 \\
\hline
\end{tabular}




\begin{tabular}{|c|c|c|c|c|c|c|}
\hline 样地 & 核桃梑胸径 & 核桃楸个数 & 海拔 & 坡度 & 坡向 & 乔木层盖度 \\
\hline Plot & Diameter $(\mathrm{cm})$ & Number & Elevation (m) & Slope & Aspect & Coverage \\
\hline TJ8 & 5.92 & 2 & 900 & 30 & 0 & 85 \\
\hline TJ9 & 12.32 & 2 & 820 & 40 & 340 & 85 \\
\hline $\mathrm{TJ} 10$ & 9.49 & 11 & 820 & 40 & 160 & 90 \\
\hline TJ11 & 3.38 & 5 & 860 & 30 & 180 & 85 \\
\hline $\mathrm{TJ} 12$ & 4.68 & 2 & 800 & 45 & 180 & 13.5 \\
\hline TJ13 & 4.78 & 1 & 750 & 15 & 90 & 80 \\
\hline TJ14 & 8.50 & 3 & 700 & 10 & 180 & 85 \\
\hline TJ15 & 5.38 & 6 & 680 & 22 & 150 & 90 \\
\hline TJ16 & 6.78 & 8 & 635 & 19 & 225 & 85 \\
\hline TJ17 & 6.59 & 13 & 630 & 19 & 210 & 85 \\
\hline $\mathrm{TJ} 18$ & 8.03 & 15 & 500 & 10 & 0 & 75 \\
\hline TJ19 & 8.03 & 3 & 500 & 20 & 165 & 75 \\
\hline TJ20 & 5.67 & 3 & 500 & 15 & 165 & 80 \\
\hline $\mathrm{TJ} 21$ & 7.68 & 11 & 420 & 30 & 180 & 80 \\
\hline $\mathrm{TJ} 22$ & 3.06 & 33 & 420 & 15 & 180 & 90 \\
\hline $\mathrm{TJ} 23$ & 7.83 & 13 & 720 & 15 & 0 & 70 \\
\hline $\mathrm{TJ} 24$ & 7.45 & 19 & 735 & 15 & 0 & 70 \\
\hline $\mathrm{TJ} 25$ & 3.85 & 13 & 340 & 18 & 270 & 70 \\
\hline TJ26 & 2.83 & 40 & 340 & 18 & 270 & 85 \\
\hline TJ27 & 5.92 & 16 & 330 & 18 & 270 & 60 \\
\hline $\mathrm{TJ} 28$ & 4.81 & 22 & 320 & 18 & 270 & 70 \\
\hline TJ29 & 2.13 & 35 & 325 & 5 & 45 & 90 \\
\hline TJ30 & 1.43 & 1 & 740 & 50 & 0 & 80 \\
\hline TJ31 & 1.62 & 2 & 720 & 40 & 315 & 20 \\
\hline $\mathrm{TJ} 32$ & 5.00 & 3 & 706 & 60 & 315 & 60 \\
\hline TJ33 & 3.34 & 1 & 705 & 45 & 315 & 80 \\
\hline TJ34 & 10.45 & 1 & 680 & 45 & 315 & 85 \\
\hline TJ35 & 9.52 & 1 & 655 & 45 & 0 & 70 \\
\hline TJ36 & 6.27 & 1 & 645 & 50 & 0 & 50 \\
\hline TJ37 & 2.74 & 1 & 640 & 45 & 0 & 85 \\
\hline TJ38 & 3.98 & 3 & 711 & 40 & 0 & 85 \\
\hline TJ39 & 7.68 & 5 & 730 & 15 & 270 & 75 \\
\hline TJ40 & 9.65 & 6 & 750 & 35 & 180 & 70 \\
\hline TJ41 & 10.86 & 1 & 816 & 25 & 180 & 85 \\
\hline TJ42 & 10.86 & 1 & 812 & 45 & 180 & 85 \\
\hline TJ43 & 9.11 & 10 & 808 & 25 & 180 & 85 \\
\hline TJ44 & 7.77 & 2 & 829 & 30 & 45 & 90 \\
\hline TJ45 & 6.82 & 2 & 810 & 40 & 0 & 75 \\
\hline TJ46 & 4.17 & 1 & 829 & 40 & 45 & 90 \\
\hline TJ47 & 5.61 & 10 & 751 & 25 & 135 & 50 \\
\hline TJ48 & 5.83 & 11 & 620 & 30 & 135 & 75 \\
\hline TJ49 & 7.96 & 6 & 561 & 15 & 135 & 60 \\
\hline
\end{tabular}


10.17521/cjpe.2018.0161-S1

\begin{tabular}{ccccccc}
\hline $\begin{array}{c}\text { 样地 } \\
\text { Plot }\end{array}$ & $\begin{array}{c}\text { 核桃楸胸径 } \\
\text { Diameter }(\mathrm{cm})\end{array}$ & $\begin{array}{c}\text { 核桃楸个数 } \\
\text { Number }\end{array}$ & $\begin{array}{c}\text { 海拔 } \\
\text { Elevation }(\mathrm{m})\end{array}$ & $\begin{array}{c}\text { 坡度 } \\
\text { Slope }\end{array}$ & $\begin{array}{c}\text { 坡向 } \\
\text { Aspect }\end{array}$ & $\begin{array}{c}\text { 乔木层盖度 } \\
\text { Coverage }\end{array}$ \\
\hline TJ50 & 5.86 & 3 & 558 & 13 & 135 & 85 \\
TJ51 & 5.80 & 18 & 558 & 5 & 135 & 85 \\
TJ52 & 7.01 & 12 & 556 & 21 & 135 & 80 \\
TJ53 & 7.17 & 2 & 562 & 15 & 135 & 85 \\
TJ54 & 7.52 & 2 & 565 & 30 & 135 & 75 \\
TJ55 & 13.38 & 1 & 567 & 20 & 135 & 85 \\
TJ56 & 5.99 & 2 & 573 & 17 & 135 & 80 \\
TJ57 & 1.62 & 1 & 570 & 30 & 135 & 80 \\
\hline
\end{tabular}

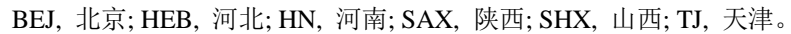

BEJ, Beijing; HEB, Hebei; HN, Henan; SAX, Shaanxi; SHX, Shanxi; TJ, Tianjin. 